\title{
Numerical and Computational Analysis of models for Stochastic activity of neurons
}

\author{
Ana Cláudia dos Reis Valentim ${ }^{1}$ \\ Laboratório Nacional de Computação Científica, LNCC, Petrópolis, RJ \\ Alexandre Loureiro Madureira ${ }^{2}$ \\ Laboratório Nacional de Computação Científica, LNCC, Petrópolis, RJ \\ Hugo de la Cruz ${ }^{3}$ \\ Fundação Getúlio Vargas, FGV, Rio de Janeiro, RJ
}

\begin{abstract}
A current issue in Computational Neuroscience is to develop models that describe the neuronal firing accurately and with low computational cost. The Hodgkin-Huxley model fulfills the first criteria but fails in the second one. In order to handle it, there are other simpler models, as the Integrate-and-fire. Both methods present deterministic approaches, and in order to obtain more accurate results, we have considered the addition of Brownian noise. We have obtained more accurate results and compared them.
\end{abstract}

Key-words. Neuroscience. Numerical Methods. Hodgkin-Huxley model. Integrate-andFire model. Stochastic Differential Equations.

\section{Introduction}

In 1952, in the paper A quantitative description of Membrane Current and its application to conduction and excitation in nerve [7], Hodgkin and Huxley presented a voltage-dependent model for neuronal firing as a nonlinear phenomenon. This model received their names and it is very uselful because it captures biological aspects, but it is difficult to solve and presents very sensitive parameters [12]. Simpler and cheaper models were developed posteriorly, aiming to obtain, e.g., firing rates [10]. Considering simplicity and low computational cost, the Integrate-and-Fire (IF) model is the most efficient among other models [9].

Both models approaches neuronal firing deterministically. That is not accurate, because it's known that, e.g., cortical and spontanous activity cells do not present this kind of behavior $[2,8]$. These irregularities are biologically caused by many factors and we represent the sum of these irregularities in the model adding white noise on it [11].

We aim to obtain more accurate results for spike trains. We also aim to compare $\mathrm{HH}$ and IF models in relation to computational cost and output probability distribution.

\footnotetext{
${ }^{1}$ anacrv@lncc.br

${ }^{2}$ alm@lncc.br

${ }^{3}$ hugo.delacruz@fgv.br
} 


\section{The Hodgkin-Huxley model}

The HH model describes action potencials through an analogy between the cell membrane and a simple electric circuit [5]. It is the most complete model so far. It considers a selectively permeable cell membrane, containing protein channels for Sodium and Potassium ions. The opening and closing process of these channels occurs stochastically, i.e., one can not estimate deterministically if the channels will be open or closed in a certain instant of time.

The mathematical model consists of a parabolic PDE and three ODEs. The first equation of the $\mathrm{HH}$ model shows how voltage varies in time and space:

$$
C \frac{\partial V}{\partial t}=\mu \frac{\partial^{2} V}{\partial x^{2}}-\sum_{i=1}^{3} g_{i}\left(V-E_{i}\right)+I_{e x t}+R_{1}
$$

The voltage rate, which is multiplied by the membrane specific capacitance $\mathrm{C}$, is the sum of the ionic currents, the spatial term and input current $I_{\text {ext }}$ disturbed by an addictive noise $R_{1}$. These ionic currents are given by Kirchhoff's law, expressed as conductance times the concentration gradient for each ion (voltage minus the Nernst potential). As we have Sodium, Potassium and and leak channels, the index $i$ corresponds to each of these, respectively. The spatial variation is multiplied by a coeficient of diffusion $\mu$, responsible for the spatial diffusion.

The other three ODEs model how the dimensionless gating variables vary in time. These variables represent the probability of opening and closing of activation gates, for Potassium ion $(m)$, and of activation or inactivation gates, for Sodium ion $(h$ and $n)$.

$$
\begin{aligned}
\frac{d m}{d t} & =(1-m) \alpha_{m}(V)-m \beta_{m}(V) ; \\
\frac{d h}{d t} & =(1-h) \alpha_{h}(V)-h \beta_{h}(V) ; \\
\frac{d n}{d t} & =(1-n) \alpha_{n}(V)-n \beta_{n}(V) .
\end{aligned}
$$

These variations are given by the convex combination of the gating variable by the gating functions, which show how the probabilities of opening and closing vary according to the voltage.

Therefore, the HH model is formed by these four coupled and nonlinear equations. We emphasize that in this paper we take $\mu=0$.

\section{The Integrate-and-fire model}

Despite the HH model being the most complete model so far, it presents some disadvantages, as discussed previously. The Integrate-and-Fire model aims to provide spike rates in a certain time interval only with the information that the neuron has reached the action threshold. This model has the same deterministic features as the HH model, so we also have added white noise to it $[3,11]$. 
The model consists of one ODE that presents a different dynamics compared to the HH model: the neuron reaches the predefined action threshold $\vartheta$, the membrane potential returns to resting potential and the process restarts [11].

$$
\tau_{m} \frac{d v(t)}{d t}=-\left(v(t)-E_{L}\right)+R I(t)+R_{2}, \quad \forall t \in[0, T], \quad T \in \mathbb{N}
$$

The time variation is multiplied by a time constant $\tau_{m}$, determined by the model of one compartment capacitance and by the average conductances of Sodium and leaky channels. We also have the concentration gradient given by the subtraction of the voltage by the resting potential $E_{L}$. In this model we also have a input current $R I(t)$ disturbed by a noise $R_{2}$. Considering $t^{f}$ to be the instant of time that the threshold is reached, we finish it considering $v\left(t^{f}\right)=\vartheta$.

There are another two ways to perturb the IF model, but the one given by equation (1) is the only that can be compared directly to the HH model. It occurs because in both cases the noise is added in the input current.

\section{Numerical Solution}

The HH system and the IF dynamic were discretized using finite difference methods. For the stochastic cases, we have used slightly different methods from the corresponding deterministic ones. The computational experiments consist of shooting a spike train in order to verify if the addition of noise reproduces its features more precisely. For this purpose, HH and IF models were used. All numerical experiments were implemented and carried out in Matlabß R2012b version.

The noisy HH model was discretized using Euler-Maruyama(EM) [6].For values less than $\Delta t=10^{-4}$, the mean and the standard deviaton (SD) become invariant. Furthermore, the SD converges to $7.92 \times 10^{-4}$. By way of example, we show a discretization with the EM Method. The noise is given by $\mathrm{dW}\left(t^{n}\right)=\sqrt{\Delta t} N(0,1)^{n}[6]$, where $\mathrm{N}(0,1)$ are independent standard Gaussian random variables.

$$
\begin{aligned}
V^{n+1} & =V^{n}+\Delta t\left[\frac{I_{e x t}-g_{N a}^{n}-g_{K}^{n}-g_{L}^{n}}{C}\right]+\sigma_{1} d W\left(t^{n}\right) ; \\
n^{n+1} & =n^{n}+\Delta t \phi\left[\alpha_{n}(V)(1-n)-\beta_{n}(V) n\right] ; \\
m^{n+1} & =m^{n}+\Delta t \phi\left[\alpha_{m}(V)(1-m)-\beta_{m}(V) m\right] ; \\
h^{n+1} & =h^{n}+\Delta t \phi\left[\alpha_{h}(V)(1-h)-\beta_{h}(V) h\right] .
\end{aligned}
$$

The initial conditions and parameters are given by [5], [4] and [1]. 
Table 1: Experimental parameters for $\mathrm{HH}$ model when $\mu=0$.

\begin{tabular}{|c|c|c|}
\hline Parameter & Meaning & Value \\
\hline$C$ & Membrane specific capacitance & $1 \mu \mathrm{F} \mathrm{cm}$ \\
\hline$E_{N a}, E_{K}, E_{L e a k}$ & Nernst potentials & $50,-77,-54.4 \mathrm{mV}$ \\
\hline $\bar{g}_{N a}, \bar{g}_{N a}, \bar{g}_{N a}$ & Maximum conductances & $120,36,0.3 \mathrm{mScm}$ \\
\hline$I_{e x t}$ & Input current & $12 \mathrm{mV}$ \\
\hline$m, n, h$ & Gating variables & $0.1,0.4,0.4$ \\
\hline$\sigma_{1}$ & Intensity constant & 24 \\
\hline
\end{tabular}

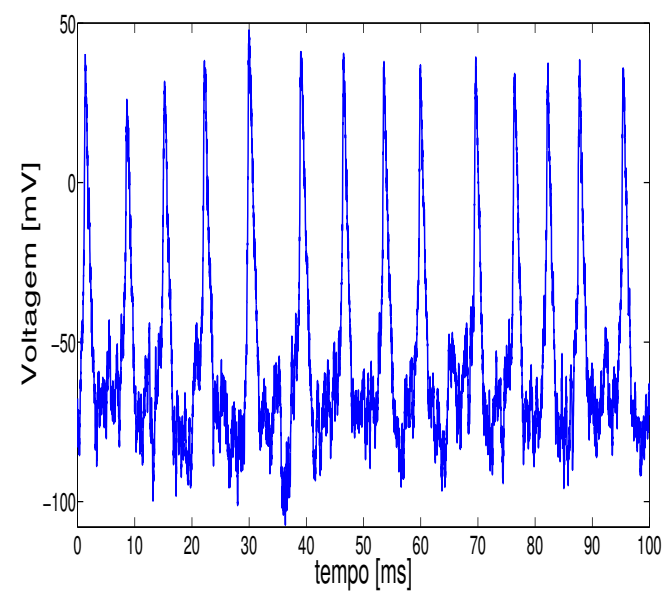

Figure 1: Spike train with white noise through EM Method for the time interval of $100 \mathrm{~ms}$.

By way of comparison to the $\mathrm{HH}$ model, here is the noisy IF discretization with the EM Method $[6,11]$.

$$
V^{n+1}=V^{n}-\frac{\Delta t}{\tau_{m}}\left(\left(V^{n}-E_{L}\right)-R I_{e x t}\right)+\sigma_{2} d W\left(t^{n}\right)
$$

The initial conditions and parameters are given by [11].

Table 2: Experimental parameters for IF model.

\begin{tabular}{|c|c|c|}
\hline Parameter & Meaning & Value \\
\hline$\tau_{m}$ & Time constant & $10 \mathrm{~ms}$ \\
\hline$E_{L}$ & Resting potential & $-65 \mathrm{mV}$ \\
\hline$\sigma_{2}$ & Intensity constant & 2 \\
\hline
\end{tabular}




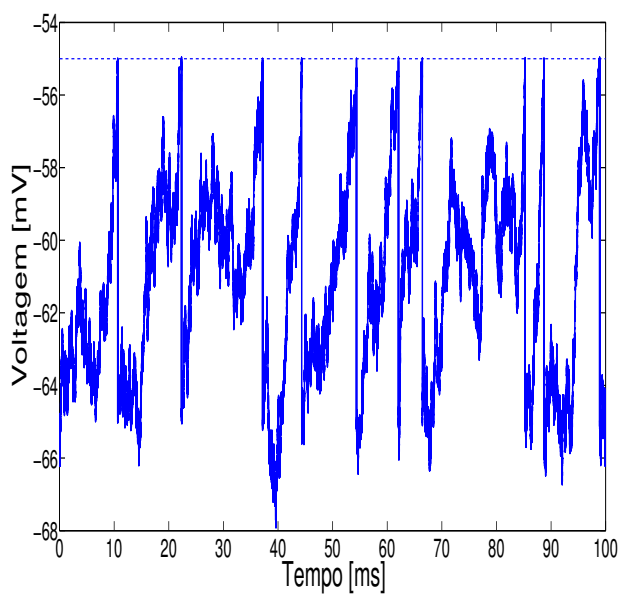

Figure 2: Subthreshold dynamic with white noise through EM Method for the time interval of $100 \mathrm{~ms}$.

It can be observed that for both cases, the addition of noise has provided a more accurate evolution of the firing neuron, by capturing its irregularities. Besides every spike having different intervals interspikes two by two in the first case for the $\mathrm{HH}$ and IF models, the action threshold is also diferent in a spike train (except in the IF case, because the threshold is fixed). The runtime for both models also was compared. Using the computer processor Intel(R) Core(TM i7 - $4790 \mathrm{CPU} @ 3.60 \mathrm{~Hz})$ in a machine with $16384340 \mathrm{kB}$ of memory, we have observed that the IF model can be more than twice as fast than the HH model for this case.

In order to analyse the output probability distribution for the case where $\mu=0$, we have made a Monte Carlo approach. It were realized 500 samples of spike trains, with 500 neuron firing each one, totalizing 250000 firings. We expect that, for non-spontaneous firings, but with Gaussian noise input, the histogram can be well approximated by a lognormal distribution $[2,11]$.

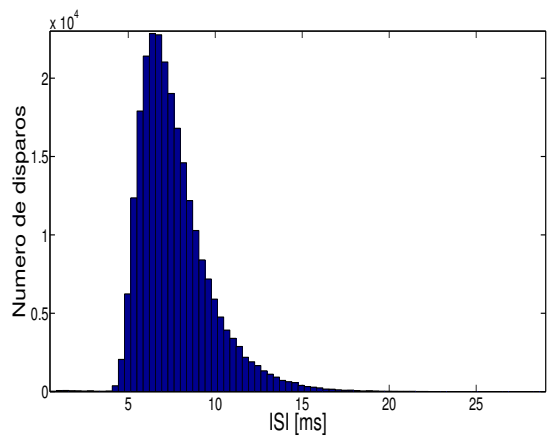

Figure 3: ISI-histogram for HH model considering 500 random samples with 500 firing each. 


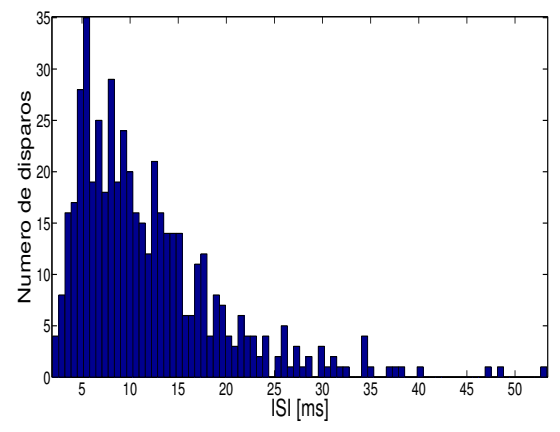

Figure 4: ISI-histogram for IF model considering 500 random samples with 500 firing each.

Table 3: Dispersion data for IF model.

\begin{tabular}{|c|c|c|c|}
\hline Model & Time step size & Mean & SD \\
\hline HH & $10^{-4}$ & 7.744 & 2.228 \\
\hline IF & $10^{-4}$ & 11.886 & 7.659 \\
\hline
\end{tabular}

Both ISI-histograms can be well aproximated by a lognormal distribution, and according to the dispersion measures we observe that the $\mathrm{HH}$ model is closer to a exponential distribution, which shows that spike trains under our computational assumption are often generated with a Poisson process [11].

\section{Concluding Remarks}

The addition of standard Brownian Motion uncertainty to the $\mathrm{HH}$ and IF models provides more accurate results. This can be observed by the fact that the stochastic model reproduces inherent shape irregularities in excitatory and inhibitory cortical cells, while the deterministic model reproduces constant firing. One can also observe that the ISIhistogram for the temporal case can be well approximated by a lognormal distribution as expected for one source of Gaussian noise.

\section{References}

[1] R. R. Borges, K. C. Iarosz, A. M. Batista, I. L. Caldas, F. S. Borges and E. L. Lameu, Sincronização de disparos em redes neuronais com plasticidade sináptica, Revista Brasileira de Ensino de Física, 37:2310-1 - 2310-9, 2015. DOI: 10.1590/S180611173721787.

[2] N. Brunel and X. Wang, Effects of Neuromodulation in a Cortical Network Model of Object Working Memory Dominated by Recurrent Inhibition, Journal of Computational Neuroscience, 11:63-85, 2001. DOI: 10.1023/A:1011204814320. 
[3] W. Gerstner, Population Dynamics of Spiking Neurons: Fast Transients, Asynchoronous States, and Locking, Neural Computation, 12:43-89, 2000. DOI: $10.1162 / 089976600300015899$.

[4] M. Hanslien, K. H. Karlsen and A. Tveito, A maximum principle for and explicit finite difference scheme approximating the Hodgkin-Huxley model, BIT Numerical Mathematics, 45:725-741, 2005. DOI: 10.1007/s10543-005-0023-2.

[5] G. Hermentrout and D. H. Terman, Mathematical Foundations of Neuroscience, Springer, 35:1-28, 2013. ISBN: 978-0-387-87707-5.

[6] D. J. Higham, An Algorithmic Introduction to Numerical Simulation of Stochastic Differential Equations, SIAM Review, 43:525-546, 2001. DOI: $10.1137 /$ S0036144500378302.

[7] A. L. Hodgkin and A. F. Huxley, A quantitative description of Membrane Current and its application to conduction and excitation in nerve, J. Physiol., 117:500-544, 1952. DOI: 10.1113/jphysiol.1952.sp004764.

[8] E. M. Izhikevich, Simple model of spiking neurons, IEEE Transactions on neural networks, 14:1569-1672, 2003. DOI: 10.1109/TNN.2003.820440.

[9] E. M. Izhikevich, Which Model to Use for Cortical Spiking Neurons?, IEEE Transactions on neural networkns, 15:1063-1070, 2004. DOI: 10.1109/TNN.2004.832719.

[10] C. Meunier and I. Seveg, Playing devil's advocate: is this Hodgkin-Huxley model useful?, TRENDS in Neuroscience, 25:558-563, 2002. DOI: 10.1016/S01662236(02)02278-6.

[11] T. P. Trappenberg, Fundamentals of Computational Neuroscience, OXFORD University Press, pages 53-71, 2010. ISBN: 978-0-19-956841-3.

[12] A. T. Valderrama, J. Witteveen, M. Navarro and J. Blom, Uncertainty Propagation in Nerve Impulses Through the Action Potential Mechanism, Journal of Mathematical Neuroscience, 5:1-9, 2015. DOI: 10.1186/2190-8567-5-3. 